\title{
Note on weakly fractional differential equations
}

\author{
Michal Fečkan ${ }^{1,2}$, Michal Pospišil $1^{1,2}$ and JinRong Wang ${ }^{3,4^{*}}$ (1)
}

${ }^{*}$ Correspondence: wjr9668@126.com

${ }^{3}$ Department of Mathematics, Guizhou University, Guiyang, China

${ }^{4}$ School of Mathematical Sciences, Qufu Normal University, Qufu, China Full list of author information is available at the end of the article

\begin{abstract}
In this paper, we compare solutions of q-order fractional differential equations of Caputo type for $q$ near 1 with solutions of the corresponding 1-order ordinary differential equations. By establishing the explicit lower and upper bounds of Mittag-Leffler functions, we obtain the effective convergence results. It is shown that the limit cases $q \rightarrow 1_{+}$and $q \rightarrow 1_{-}$are different. A simple illustrative example is also presented.
\end{abstract}

Keywords: Weakly fractional differential equation; Caputo fractional derivative; Comparison; Mittag-Leffler function

\section{Introduction}

Fractional differential equations (FDEs) are a rapidly developing area of mathematics with many stimulating applications [1-4]. Recently, plenty interesting existence and controllability results on the theory of solutions of FDEs or fractional inclusions have been given in [5-22]. Mathematical modeling approaches using fractional derivatives are presented in [17-22] with numerical simulations on various challenging topics.

On the one hand, several properties of ordinary or partial differential equations (DEs) appear in FDEs as well, like asymptotic properties of solutions or equilibria. On the other hand, unlike to DEs, FDEs have no nonconstant periodic solutions and they do not create dynamical systems, which is one of the most obvious characteristics in studying FDEs. So there is a natural question to study the relationship between solutions of FDEs and DEs when the order $q$ of FDEs is near to a natural number $n \in \mathbb{N}$. Here, we call such FDEs weakly fractional, which can be used to seek numerically the solutions of DEs.

In this paper, we investigate for simplicity the case when $q$ is near to $n=1$, but our method can be directly extended to any $n$. We study two cases: $q \rightarrow 1_{-}$in Sect. 2 and $q \rightarrow 1_{+}$in Sect. 3. We derive error estimates in both cases. A simple numerical illustrative example is given to demonstrate theoretical results. Our next step will be to extend this paper for weakly fractional semilinear evolution equations in Banach spaces.

\section{The case $q \rightarrow 1$}

Consider a fractional differential equation

$$
\begin{aligned}
& D_{0}^{q} x(t)=f(t, x(t)), \quad t \in \mathbb{R}_{+}=[0, \infty), \\
& x(0)=x_{0}
\end{aligned}
$$

(c) The Author(s) 2019. This article is distributed under the terms of the Creative Commons Attribution 4.0 International License (http://creativecommons.org/licenses/by/4.0/), which permits unrestricted use, distribution, and reproduction in any medium, provided you give appropriate credit to the original author(s) and the source, provide a link to the Creative Commons license, and indicate if changes were made. 
where $D_{0}^{q}$ is the Caputo fractional derivative of order $q \in(0,1)$ with the lower limit at zero,

$$
D_{0}^{q} x(t)=\frac{1}{\Gamma(1-q)} \frac{d}{d t} \int_{0}^{t}(t-s)^{-q}(x(s)-x(0)) d s,
$$

and $f \in C\left(\mathbb{R}_{+} \times \mathbb{R}^{n}, \mathbb{R}^{n}\right)$ along with an ordinary differential equation

$$
\begin{aligned}
& y^{\prime}(t)=f(t, y(t)), \quad t \in \mathbb{R}_{+}, \\
& y(0)=y_{0},
\end{aligned}
$$

where $x_{0}, y_{0} \in \mathbb{R}^{n}$. We suppose

(H) There are nonnegative constants $M$ and $L$ such that $\|f(t, x)\| \leq M$ and $\|f(t, x)-f(t, y)\| \leq L\|x-y\|$ for any $t \in \mathbb{R}_{+}$and $x, y \in \mathbb{R}^{n}$, where $\|\cdot\|$ is a norm on $\mathbb{R}^{n}$.

It is well known [4] that problem (1) is equivalent to the following integral equation:

$$
x(t)=x_{0}+\frac{1}{\Gamma(q)} \int_{0}^{t}(t-s)^{q-1} f(s, x(s)) d s .
$$

Then we derive

$$
\begin{aligned}
\| x(t) & -y(t) \| \\
\leq & \left\|x_{0}-y_{0}\right\|+\frac{1}{\Gamma(q)} \int_{0}^{t}(t-s)^{q-1}\|f(s, x(s))-f(s, y(s))\| d s \\
& \quad+\int_{0}^{t}\left|1-\frac{1}{\Gamma(q)}(t-s)^{q-1}\right|\|f(s, y(s))\| d s \\
\leq & \left\|x_{0}-y_{0}\right\|+\frac{L}{\Gamma(q)} \int_{0}^{t}(t-s)^{q-1}\|x(s)-y(s)\| d s+M \int_{0}^{t}\left|1-\frac{1}{\Gamma(q)}(t-s)^{q-1}\right| d s \\
= & \left\|x_{0}-y_{0}\right\|+\frac{L}{\Gamma(q)} \int_{0}^{t}(t-s)^{q-1}\|x(s)-y(s)\| d s+M \int_{0}^{t}\left|1-\frac{1}{\Gamma(q)} s^{q-1}\right| d s .
\end{aligned}
$$

Thus by the Henry-Gronwall inequality (see [23, Corollary 2]), we get

$$
\|x(t)-y(t)\| \leq\left(\left\|x_{0}-y_{0}\right\|+M \int_{0}^{t}\left|1-\frac{1}{\Gamma(q)} s^{q-1}\right| d s\right) E_{q}\left(L t^{q}\right)
$$

for any $t \in \mathbb{R}_{+}$, where $E_{q}$ is the Mittag-Leffler function [24]. We continue with the case $x_{0}=y_{0}$. Then we get

$$
\|x(t)-y(t)\| \leq M \theta_{q}(t), \quad \theta_{q}(t):=\int_{0}^{t}\left|1-\frac{1}{\Gamma(q)} s^{q-1}\right| d s E_{q}\left(L t^{q}\right)
$$

for any $t \in \mathbb{R}_{+}$. The equation

$$
1-\frac{1}{\Gamma(q)} s^{q-1}=0
$$

has the only solution $s_{0}>0$ given by

$$
s_{0}=s_{0}(q)=\Gamma(q)^{\frac{1}{q-1}} .
$$


Note that the function $s_{0}(q)$ is increasing on $(0,1)$ with $\lim _{q \rightarrow 0_{+}} s_{0}(q)=0$ and

$$
\lim _{q \rightarrow 1_{-}} s_{0}(q)=e^{\lim _{q \rightarrow 1_{-}} \frac{\ln [\Gamma[q]]}{q-1}}=e^{\lim _{q \rightarrow 1-} \frac{\Gamma^{\prime}[q]}{\Gamma[q]}}=e^{-\gamma} \doteq 0.561459
$$

for the Euler constant $\gamma$. Next, clearly, we have

$$
1-\frac{1}{\Gamma(q)} s^{q-1} \begin{cases}<0 & \text { for } 0<s<s_{0} \\ =0 & \text { for } s=s_{0} \\ >0 & \text { for } s>s_{0} .\end{cases}
$$

Consequently, we obtain

$$
\int_{0}^{t}\left|1-\frac{1}{\Gamma(q)} s^{q-1}\right| d s= \begin{cases}\frac{t^{q}}{\Gamma(q+1)}-t & \text { for } 0<t<s_{0} \\ \lambda(q):=\frac{\Gamma(q)^{\frac{q}{q-1}}}{\Gamma(q+1)}-\Gamma(q)^{\frac{1}{q-1}} & \text { for } t=s_{0} \\ \frac{-t^{q}+t \Gamma(q+1)+2 \Gamma(q)^{\frac{q}{q-1}}}{\Gamma(q+1)}-2 \Gamma(q)^{\frac{1}{q-1}} & \text { for } t>s_{0} .\end{cases}
$$

We can check numerically that $\lambda^{\prime \prime}(q)>0$ for $q \in(0,1)$, then that $\lambda^{\prime}(q)$ is increasing from $-\infty$ to $-e^{-\gamma} \doteq-0.561459$, and then that $\lambda(q)$ is decreasing from 1 to 0 . So we consider $q \in(1 / 2,1)$ and then $-0.751988 \leq \lambda^{\prime}(q) \leq-0.561459$. This implies that

$$
0<\lambda(q) \leq 0.8(1-q)
$$

for $q \in(1 / 2,1)$. Next, by [25, Lemma 2], we have the following.

Lemma 2.1 For all $t \in \mathbb{R}_{+}, q \in(0,1)$, and $\kappa>0$, it holds

$$
1 \leq E_{q}\left(\kappa t^{q}\right) \leq \frac{e^{\kappa^{\frac{1}{q}}} t}{q}
$$

Furthermore, (5) implies

$$
\int_{0}^{t}\left|1-\frac{1}{\Gamma(q)} s^{q-1}\right| d s \leq\left|t-\frac{t^{q}}{\Gamma(q+1)}\right|+2 \lambda(q)
$$

for $t \in \mathbb{R}_{+}$. So if $q \in(1 / 2,1)$, then by Lemma 2.1 we get

$$
\theta_{q}(t) \leq \frac{e^{L^{\frac{1}{q}}} t}{q}\left(\left|t-\frac{t^{q}}{\Gamma(q+1)}\right|+2 \lambda(q)\right) \leq 2 e^{t \tilde{L}}\left(\left|t-\frac{t^{q}}{\Gamma(q+1)}\right|+2 \lambda(q)\right)
$$

for $\tilde{L}=\max \left\{L, L^{2}\right\}$.

Now we are ready to deal with (3). First, (3) immediately implies the following expected result.

Theorem 2.2 Under assumption $(\mathrm{H})$, the solution $x(t)$ of $(1)$ uniformly converges on any finite interval $[0, T], T>0$, of $\mathbb{R}_{+}$to the solution $y(t)$ of $(2)$ if $q \rightarrow 1_{-}$and $x_{0}=y_{0}$. 
Proof The proof follows directly from (3), (5) and by

$$
\lim _{q \rightarrow 1_{-}}\left|t-\frac{t^{q}}{\Gamma(q+1)}\right|=0
$$

uniformly for $t \in[0, T]$ and any fixed $T>0$.

Next, we take any $\epsilon>0$ and consider an equation

$$
\theta_{q}(t)=\epsilon
$$

Clearly, $\theta_{q}(t)$ is increasing on $\mathbb{R}_{+}$from 0 to $\infty$. Thus (8) has the only solution $\bar{t}(\epsilon, q) \in \mathbb{R}_{+}$. By the above observations we can easily see that $\lim _{\epsilon \rightarrow 0_{+}} \bar{t}(\epsilon, q)=0$ and $\lim _{q \rightarrow 1_{-}} \bar{t}(\epsilon, q)=\infty$.

Furthermore, the function $t \mapsto t-\frac{t^{q}}{\Gamma(q+1)}$ is nonpositive on $\left[0, r_{0}\right]$ and nonnegative on $\left[r_{0}, \infty\right)$ for

$$
r_{0}=r_{0}(q)=\Gamma(q+1)^{\frac{1}{q-1}} .
$$

Note that the function $r_{0}(q)$ is increasing on $(0,1)$ from $\lim _{q \rightarrow 0_{+}} r_{0}(q)=1$ to $\lim _{q \rightarrow 1-} r_{0}(q)=$ $e^{1-\gamma} \doteq 1.526205$.

Next, we study the function $\phi_{t}(q):=\frac{t^{q}}{\Gamma(q+1)}$ on $(0,1)$ for $t>0$. We have

$$
\phi_{t}^{\prime}(q)=\frac{t^{q} \ln t}{\Gamma(q+1)}-\frac{t^{q} \Gamma^{\prime}(q+1)}{\Gamma^{2}(q+1)} .
$$

For $t \in(0,1]$ and $q \in(1 / 2,1)$, we get

$$
\left|\phi_{t}^{\prime}(q)\right| \leq-\frac{\sqrt{t} \ln t}{\Gamma(q+1)}+\left|\frac{\Gamma^{\prime}(q+1)}{\Gamma^{2}(q+1)}\right| \leq 1.253
$$

while for $1 \leq t \leq T$ and $q \in(1 / 2,1)$, we get

$$
\left|\phi_{t}^{\prime}(q)\right| \leq 1.12838 T^{q} \ln T+0.422784 T^{q}
$$

for $T>1$. Consequently, we have

$$
\left|\phi_{t}^{\prime}(q)\right| \leq 1.253+1.12838 T^{q} \ln T+0.422784 T^{q}
$$

for $t \in(0, T], T>1$, and $q \in(1 / 2,1)$. This implies

$$
\left|t-\frac{t^{q}}{\Gamma(q+1)}\right|=\left|\phi_{t}(1)-\phi_{t}(q)\right| \leq\left(1.253+1.12838 T^{q} \ln T+0.422784 T^{q}\right)(1-q)
$$

for $t \in[0, T], T>1$, and $q \in(1 / 2,1)$. Using (6), (7), and (11), we arrive at

$$
\theta_{q}(t) \leq 2 e^{T \tilde{L}}\left(3+2 T^{q} \ln T+T^{q}\right)(1-q)
$$

for $t \in[0, T], T>1$, and $q \in(1 / 2,1)$. Now, we consider instead of (8) the following one:

$$
\eta_{L, q}(T):=2 e^{T \tilde{L}}\left(3+2 T^{q} \ln T+T^{q}\right)=\frac{1}{\sqrt{1-q}} .
$$


The function $\eta_{L, q}(T)$ is increasing from $8 e^{\tilde{L}}$ to $\infty$ on $[1, \infty)$. So, for any

$$
q>1-\frac{1}{64 e^{2 \tilde{L}}}, \quad q \in(1 / 2,1)
$$

(12) has a unique solution $T_{L}(q)>1$. Note

$$
\lim _{q \rightarrow 1_{-}} T_{L}(q)=\infty
$$

Summarizing, we have the following result.

Theorem 2.3 Under assumption $(\mathrm{H})$ and for any q fulfilling (13), the solutions $x(t)$ and $y(t)$ of (1) and (2) with $x_{0}=y_{0}$, respectively, satisfy

$$
\|x(t)-y(t)\| \leq M \sqrt{1-q}
$$

for any $t \in\left[0, T_{L}(q)\right]$, where $T_{L}(q)>1$ is the unique solution of (12).

\section{The case $q \rightarrow 1_{+}$}

Consider a fractional differential equation

$$
\begin{aligned}
& D_{0}^{q} x(t)=f(t, x(t)), \quad t \in \mathbb{R}_{+}, \\
& x(0)=x_{0}, \\
& x^{\prime}(0)=x_{1},
\end{aligned}
$$

where $q \in(1,2)$ and $f \in C\left(\mathbb{R}_{+} \times \mathbb{R}^{n}, \mathbb{R}^{n}\right)$ along with an ordinary differential equation

$$
\begin{aligned}
& y^{\prime}(t)=f(t, y(t))+y_{1}, \quad t \in \mathbb{R}_{+}, \\
& y(0)=y_{0},
\end{aligned}
$$

where $x_{0}, x_{1}, y_{0}, y_{1} \in \mathbb{R}^{n}$. Again, we suppose assumption (H). It is known [2, Theorem 3.24] that initial value problem (15) is equivalent to the integral equation

$$
x(t)=x_{0}+x_{1} t+\frac{1}{\Gamma(q)} \int_{0}^{t}(t-s)^{q-1} f(s, x(s)) d s
$$

Analogously to the previous section, we derive

$$
\begin{aligned}
\|x(t)-y(t)\| \leq & \left\|x_{0}-y_{0}\right\|+\left\|x_{1}-y_{1}\right\| t \\
& +\frac{L}{\Gamma(q)} \int_{0}^{t}(t-s)^{q-1}\|x(s)-y(s)\| d s+M \int_{0}^{t}\left|1-\frac{1}{\Gamma(q)} s^{q-1}\right| d s,
\end{aligned}
$$

and the Henry-Gronwall inequality yields

$$
\|x(t)-y(t)\| \leq\left(\left\|x_{0}-y_{0}\right\|+\left\|x_{1}-y_{1}\right\| t+M \int_{0}^{t}\left|1-\frac{1}{\Gamma(q)} s^{q-1}\right| d s\right) E_{q}\left(L t^{q}\right) .
$$


Hence, for $x_{0}=y_{0}, x_{1}=y_{1}$, estimation (3) follows for any $t \in \mathbb{R}_{+}$. Function $s_{0}(q)$ of (4) is increasing on $(1,2)$ from

$$
\lim _{q \rightarrow 1_{+}} s_{0}(q)=e^{\lim _{q \rightarrow 1_{+}} \frac{\ln [\Gamma[q]]}{q-1}}=e^{\lim _{q \rightarrow 1_{+}} \frac{\Gamma^{\prime}[q]}{\Gamma[q]}}=e^{-\gamma} \doteq 0.561459
$$

to 1 . So this time,

$$
1-\frac{1}{\Gamma(q)} s^{q-1} \begin{cases}>0 & \text { for } 0<s<s_{0} \\ =0 & \text { for } s=s_{0} \\ <0 & \text { for } s>s_{0}\end{cases}
$$

Consequently, we have (compare with (5))

$$
\int_{0}^{t}\left|1-\frac{1}{\Gamma(q)} s^{q-1}\right| d s= \begin{cases}t-\frac{t^{q}}{\Gamma(q+1)} & \text { for } 0<t<s_{0} \\ -\lambda(q) & \text { for } t=s_{0} \\ \frac{t^{q}-t \Gamma(q+1)-2 \Gamma(q) q^{\frac{q}{q-1}}}{\Gamma(q+1)}+2 \Gamma(q)^{\frac{1}{q-1}} & \text { for } t>s_{0}\end{cases}
$$

where $\lambda(q)$ is given by (5). One can check numerically that $-\lambda^{\prime \prime}(q)<0$ for $q \in(1,2)$. So, $-\lambda^{\prime}(q)$ is decreasing from $e^{-\gamma} \doteq 0.561459$ to $\frac{3}{4}-\frac{\gamma}{2} \doteq 0.461392$, and $-\lambda(q)$ is increasing from 0 to $1 / 2$. Hence for $q \in(1,2)$, we can estimate

$$
0 \leq-\lambda(q) \leq 0.6(q-1)
$$

Next, we need the following analog to Lemma 2.1 .

Lemma 3.1 For all $t \in \mathbb{R}_{+}, q \in(1,4 / 3)$, and $\kappa>0$, it holds

$$
1 \leq E_{q}\left(\kappa t^{q}\right) \leq \frac{e^{\kappa^{\frac{1}{q}}} t}{q}+\frac{4 \sqrt{3} \sin \frac{\pi q}{2}}{9 q}
$$

Proof Using Dzherbashyan's recursion formula [26],

$$
E_{\alpha, \beta}(z)=\frac{1}{m} \sum_{h=0}^{m-1} E_{\frac{\alpha}{m}, \beta}\left(z^{\frac{1}{m}} e^{\frac{2 \pi \imath h}{m}}\right)
$$

for $\alpha, \beta>0, z \in \mathbb{R}, m \in \mathbb{N}$, where $\imath=\sqrt{-1}$, we can write

$$
E_{q}(z)=\frac{E_{\frac{q}{2}}(\sqrt{z})+E_{\frac{q}{2}}(-\sqrt{z})}{2}
$$

for any $z>0$. Next, from [27, Theorem 2.1] we know

$$
E_{\alpha}(z)=\frac{-z \sin \pi \alpha}{\pi \alpha} \int_{0}^{\infty} \frac{e^{-r^{\frac{1}{\alpha}}} d r}{r^{2}-2 r z \cos \pi \alpha+z^{2}}
$$


for any $\alpha>0, z<0$. So, using $\cos \frac{\pi q}{2} \geq-1 / 2$ for $q \in(1,4 / 3)$, we get

$$
\begin{aligned}
E_{\frac{q}{2}}(-\sqrt{z}) & =\frac{2 \sqrt{z} \sin \frac{\pi q}{2}}{\pi q} \int_{0}^{\infty} \frac{e^{-r^{\frac{2}{q}}} d r}{r^{2}+2 r \sqrt{z} \cos \frac{\pi q}{2}+z} \\
& \leq \frac{2 \sqrt{z} \sin \frac{\pi q}{2}}{\pi q} \int_{0}^{\infty} \frac{d r}{r^{2}-r \sqrt{z}+z}=\frac{2 \sqrt{z} \sin \frac{\pi q}{2}}{\pi q} \frac{4 \sqrt{3} \pi}{9 \sqrt{z}}=\frac{8 \sqrt{3} \sin \frac{\pi q}{2}}{9 q} .
\end{aligned}
$$

Finally, applying this estimation and Lemma 2.1 to (19) results in

$$
E_{q}\left(\kappa t^{q}\right) \leq \frac{1}{2}\left(\frac{2 e^{\kappa^{\frac{1}{q}} t}}{q}+\frac{8 \sqrt{3} \sin \frac{\pi q}{2}}{9 q}\right)=\frac{e^{\kappa^{\frac{1}{q}} t}}{q}+\frac{4 \sqrt{3} \sin \frac{\pi q}{2}}{9 q} .
$$

Since by (17),

$$
\int_{0}^{t}\left|1-\frac{1}{\Gamma(q)} s^{q-1}\right| d s \leq\left|t-\frac{t^{q}}{\Gamma(q+1)}\right|-2 \lambda(q)
$$

for all $t \in \mathbb{R}_{+}$, Lemma 3.1 implies

$$
\begin{aligned}
\theta_{q}(t) & \leq\left(\frac{e^{L^{\frac{1}{q}}} t}{q}+\frac{4 \sqrt{3} \sin \frac{\pi q}{2}}{9 q}\right)\left(\left|t-\frac{t^{q}}{\Gamma(q+1)}\right|-2 \lambda(q)\right) \\
& \leq\left(e^{t \bar{L}}+\frac{4 \sqrt{3}}{9}\right)\left(\left|t-\frac{t^{q}}{\Gamma(q+1)}\right|-2 \lambda(q)\right)
\end{aligned}
$$

for $q \in(1,4 / 3)$, where $\bar{L}=\max \left\{L, L^{3 / 4}\right\}$. So we obtain a result on the uniform convergence.

Theorem 3.2 Under assumption $(\mathrm{H})$, the solution $x(t)$ of $(15)$ uniformly converges on any finite interval $[0, T], T>0$, of $\mathbb{R}_{+}$to the solution $y(t)$ of $(16)$ if $q \rightarrow 1_{+}$and $x_{0}=y_{0}, x_{1}=y_{1}$.

Proof The statement can be proved analogously to Theorem 2.2.

Next, we consider equation (8) for an arbitrary $\epsilon>0$ and $q \in(1,4 / 3)$. Clearly, $\theta_{q}(t)$ is increasing on $\mathbb{R}_{+}$from 0 to $\infty$, implying that (8) has the only solution $\bar{t}(\epsilon, q) \in \mathbb{R}_{+}$for which $\lim _{\epsilon \rightarrow 0_{+}} \bar{t}(\epsilon, q)=0$ and $\lim _{q \rightarrow 1_{+}} \bar{t}(\epsilon, q)=\infty$ hold. Moreover, the function $t \mapsto t-\frac{t^{q}}{\Gamma(q+1)}$ is nonnegative on $\left[0, r_{0}\right]$ and nonpositive on $\left[r_{0}, \infty\right)$ for $r_{0}$ given by (9). Note that $r_{0}(q)$ is increasing on $(1, \infty)$ from $\lim _{q \rightarrow 1_{+}} r_{0}(q)=e^{1-\gamma} \doteq 1.526205$ to $\infty$.

Next, we consider the function $\phi_{t}(q):=\frac{t^{q}}{\Gamma(q+1)}$ on $(1,4 / 3)$ for $t>0$. From (10), we obtain

$$
\left|\phi_{t}^{\prime}(q)\right| \leq-\frac{t \ln t}{\Gamma(q+1)}+\frac{\Gamma^{\prime}(q+1)}{\Gamma^{2}(q+1)} \leq-t \ln t+\frac{\Gamma^{\prime}\left(\frac{7}{3}\right)}{\Gamma^{2}\left(\frac{7}{3}\right)} \leq 1.038041
$$

for $t \in(0,1]$, and

$$
\left|\phi_{t}^{\prime}(q)\right| \leq T^{q} \ln T+0.51902 T^{q}
$$

for $t \in(1, T], T>1$. As a consequence, we have

$$
\left|\phi_{t}^{\prime}(q)\right| \leq 1.038041+T^{q} \ln T+0.51902 T^{q}
$$


for all $t \in(0, T], T>1, q \in(1,4 / 3)$. This implies

$$
\left|t-\frac{t^{q}}{\Gamma(q+1)}\right|=\left|\phi_{t}(1)-\phi_{t}(q)\right| \leq\left(1.038041+T^{q} \ln T+0.51902 T^{q}\right)(q-1)
$$

for $t \in(0, T], T>1, q \in(1,4 / 3)$. Using (18), (20), and (21), we arrive at

$$
\theta_{q}(t) \leq\left(e^{T \bar{L}}+\frac{4 \sqrt{3}}{9}\right)\left(3+T^{q} \ln T+T^{q}\right)(q-1)
$$

for $t \in(0, T], T>1, q \in(1,4 / 3)$. Now, we consider the equation

$$
\mu_{L, q}(T):=\left(e^{T \bar{L}}+\frac{4 \sqrt{3}}{9}\right)\left(3+T^{q} \ln T+T^{q}\right)=\frac{1}{\sqrt{q-1}} .
$$

The function $\mu_{L, q}(T)$ is increasing from $4\left(e^{\bar{L}}+4 \sqrt{3} / 9\right)$ to $\infty$ on $[1, \infty)$. So, for any

$$
q<1+\frac{1}{16\left(e^{\bar{L}}+\frac{4 \sqrt{3}}{9}\right)^{2}}, \quad q \in(1,4 / 3)
$$

(22) has a unique solution $T_{L}(q)>1$. Note that $\lim _{q \rightarrow 1_{+}} T_{L}(q)=\infty$. Summarizing, we have the following result.

Theorem 3.3 Under assumption $(\mathrm{H})$ and for any $q$ fulfilling (23), the solutions $x(t)$ and $y(t)$ of (15) and (16) with $x_{0}=y_{0}, x_{1}=y_{1}$, respectively, satisfy

$$
\|x(t)-y(t)\| \leq M \sqrt{q-1}
$$

for any $t \in\left[0, T_{L}(q)\right]$, where $T_{L}(q)>1$ is the unique solution of (22).

Next, we present a simple example illustrating the convergence results when the order $q$ is close to 1 .

Example 3.4 Let us consider the following initial-value problems:

$$
\begin{aligned}
& D_{0}^{q} x(t)=p x(t), \quad t \in \mathbb{R}_{+}, \\
& x(0)=x_{0}, \\
& y^{\prime}(t)=p y(t), \quad t \in \mathbb{R}_{+}, \\
& y(0)=y_{0}, \\
& D_{0}^{q} u(t)=p u(t), \quad t \in \mathbb{R}_{+}, \\
& u(0)=u_{0}, \\
& u^{\prime}(0)=u_{1}, \\
& v^{\prime}(t)=p v(t)+v_{1}, \quad t \in \mathbb{R}_{+}, \\
& v(0)=v_{0},
\end{aligned}
$$


Figure 1 Convergence of solutions of Caputo fractional DEs (25) (dashed blue), (27) (dot-dashed red) to solutions of ODEs (26), (28), respectively. The closer $q \in\{0.6,0.7,0.8,0.9,1.1,1.2,1.3,1.4\}$ is to 1 , the more saturated the colors are

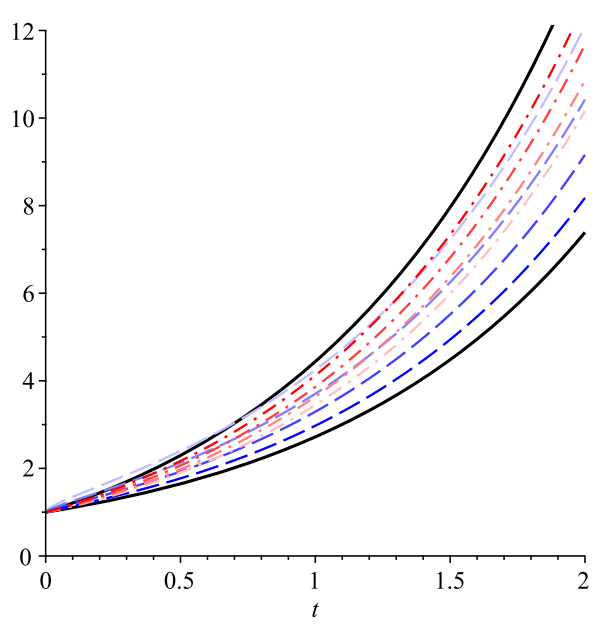

where $q \in(0,1)$ in $(25)$ and $q \in(1,2)$ in (27). The ODEs have the solutions $y(t)=y_{0} e^{p t}$, $v(t)=e^{p t}\left(v_{0}+v_{1} / p\right)-v_{1} / p$. From [2, Theorem 4.3], the other solutions are $x(t)=x_{0} E_{q}\left(p t^{q}\right)$ and $u(t)=u_{0} E_{q}\left(p t^{q}\right)+u_{1} t E_{q, 2}\left(p t^{q}\right)$. To see the convergence, we set all the initial conditions and the parameter equal to 1, i.e., $x_{0}=y_{0}=u_{0}=v_{0}=u_{1}=v_{1}=p=1$. Figure 1 depicts the convergences $x \rightarrow y$ and $u \rightarrow v$ as $q \rightarrow 1_{-}$and $q \rightarrow 1_{+}$, respectively.

The physical significance of Fig. 1 relies on demonstration of transition of $q$ through 1 . Since (25) is a one-dimensional system depending just on $x_{0}$, its limit (26) is also onedimensional. But passing to (27), we get a two-dimensional system depending on $u_{0}$ and $u_{1}$. Then its limit (28) as $q \rightarrow 1_{+}$is also two-dimensional. This makes the difference. Note that (28) is equivalent to a second order ODE

$$
\begin{aligned}
& v^{\prime \prime}(t)=p v^{\prime}(t), \quad t \in \mathbb{R}_{+}, \\
& v(0)=v_{0}, \\
& v^{\prime}(0)=\tilde{v}_{1}:=p v_{0}+v_{1} .
\end{aligned}
$$

The above arguments are more visible for $p<0$. Then by [27, Formula (7)] we see that solutions of (25), (26), and (27) asymptotically tend to zero, while the one of (28) tends to $-\frac{v_{1}}{p}$. So all these equations are dissipative. But the limit of (27) as $q \rightarrow 2_{-}$is

$$
\begin{aligned}
& z^{\prime \prime}(t)=p z(t), \quad t \in \mathbb{R}_{+}, \\
& z(0)=z_{0}, \\
& z^{\prime}(0)=z_{1},
\end{aligned}
$$

which has all solutions oscillating for $p<0$. Consequently, the dissipation of (25)-(28) is changing to oscillation on finite intervals as $q \rightarrow 2_{-}$. This is presented in Figs. 2 and 3.

These figures also support the fact that comparison estimates can be done in general only on finite intervals.

\section{Conclusion}

Solutions of $q$-order fractional differential equations of Caputo type for $q$ near 1 are compared to solutions of the corresponding 1-order ordinary differential equations, by estab- 


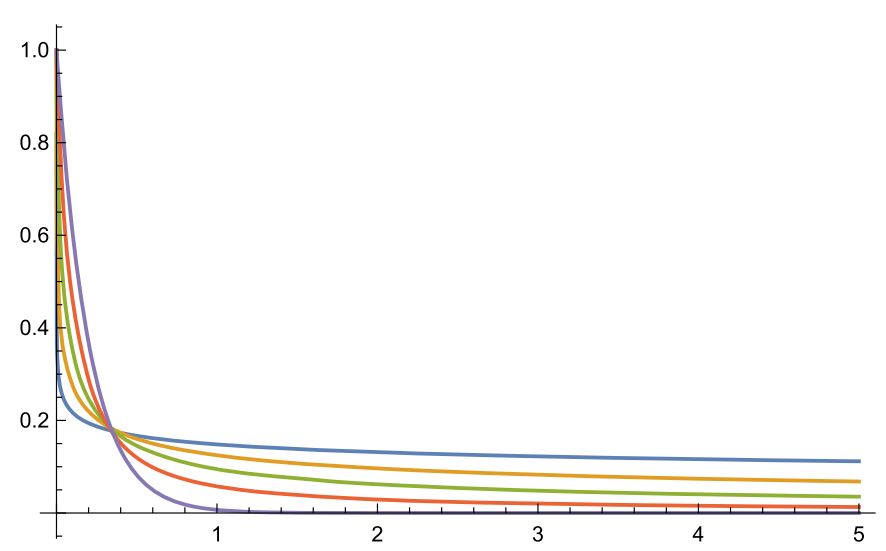

Figure 2 The solutions of Caputo fractional DEs (25) for $q \in\{0.2,0.4,0.6,0.8\}$ and (26) for $p=-5$ and $x_{0}=y_{0}=1$

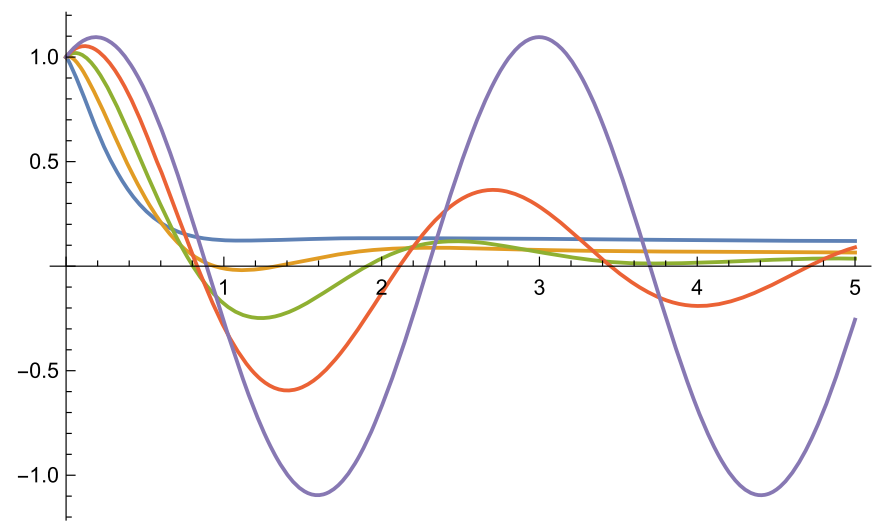

Figure 3 The solutions of Caputo fractional DEs (27) for $q \in\{1.2,1.4,1.6,1.8\}$ and (29) for $p=-5$ and $u_{0}=z_{0}=u_{1}=z_{1}=1$

lishing the effective convergence results. As a result we get that the limit cases $q \rightarrow 1_{+}$ and $q \rightarrow 1_{-}$are different. Theoretical results are demonstrated on a simple illustrative example. Our method can be directly extended to any order $q$ near a natural number.

\section{Acknowledgements}

The authors are grateful to the referees for their careful reading of the manuscript and their valuable comments.

\section{Funding}

This work is supported by the National Natural Science Foundation of China (11661016), Training Object of High Level and Innovative Talents of Guizhou Province ((2016)4006), Science and Technology Program of Guizhou Province ([2017]5788-10), Major Research Project of Innovative Group in Guizhou Education Department ([2018]012), the Slovak Research and Development Agency under the contract No. APVV-14-0378, and the Slovak Grant Agency VEGA No. 2/0153/16 and No. 1/0078/17

\section{Availability of data and materials}

Not applicable.

\section{Competing interests}

The authors declare that they have no competing interests.

\section{Authors' contributions}

All authors contributed equally to this paper. All authors read and approved the final manuscript. 


\section{Author details}

'Department of Mathematical Analysis and Numerical Mathematics, Faculty of Mathematics, Physics and Informatics, Comenius University in Bratislava, Bratislava, Slovakia. ${ }^{2}$ Mathematical Institute, Slovak Academy of Sciences, Bratislava, Slovakia. ${ }^{3}$ Department of Mathematics, Guizhou University, Guiyang, China. ${ }^{4}$ School of Mathematical Sciences, Qufu Normal University, Qufu, China.

\section{Publisher's Note}

Springer Nature remains neutral with regard to jurisdictional claims in published maps and institutional affiliations.

Received: 7 February 2019 Accepted: 8 April 2019 Published online: 18 April 2019

\section{References}

1. Fečkan, M., Wang, J.R., Pospíšil, M.: Fractional-Order Equations and Inclusions. de Gruyter, Berlin (2017)

2. Kilbas, A.A., Srivastava, H.M., Trujillo, J.J.: Theory and Applications of Fractional Differential Equations. North-Holland Mathematics Studies, vol. 204. Elsevier, Amsterdam (2006)

3. Li, C., Zeng, F.: Numerical Methods for Fractional Calculus. CRC Press, Boca Raton (2015)

4. Zhou, Y.: Fractional Evolution Equations and Inclusions: Analysis and Control. Academic Press, San Diego (2016)

5. Wang, Y., Liu, L., Wu, Y.: Positive solutions for a nonlocal fractional differential equation. Nonlinear Anal. 74, 3599-3605 (2011)

6. Zhang, X., Liu, L., Wu, Y.: Existence results for multiple positive solutions of nonlinear higher order perturbed fractional differential equations with derivatives. Appl. Math. Comput. 219, 1420-1433 (2012)

7. Wang, Y., Liu, L., Zhang, X., Wu, Y.: Positive solutions of a fractional semipositone differential system arising from the study of HIV infection models. Appl. Math. Comput. 258, 312-324 (2015)

8. Li, M., Wang, J.: Exploring delayed Mittag-Leffler type matrix functions to study finite time stability of fractional delay differential equations. Appl. Math. Comput. 324, 254-265 (2018)

9. Wang, J., Ibrahim, A.G., O'Regan, D.: Topological structure of the solution set for fractional non-instantaneous impulsive evolution inclusions. J. Fixed Point Theory Appl. 20(2), Article ID 59 (2018)

10. Wang, J., Ibrahim, A.G., O'Regan, D.: Hilfer type fractional differential switched inclusions with noninstantaneous impulsive and nonlocal conditions. Nonlinear Anal., Model. Control 23(6), 921-941 (2018)

11. Li, M., Debbouche, A., Wang, J.: Relative controllability in fractional differential equations with pure delay. Math. Methods Appl. Sci. 41, 8906-8914 (2018)

12. Zhang, X., Mao, C., Liu, L., Wu, Y.: Exact iterative solution for an abstract fractional dynamic system model for bioprocess. Qual. Theory Dyn. Syst. 16, 205-222 (2017)

13. Zhang, X., Liu, L., Wu, Y., Wiwatanapataphee, B.: Nontrivial solutions for a fractional advection dispersion equation in anomalous diffusion. Appl. Math. Lett. 66, 1-8 (2017)

14. Jiang, J., Liu, L., Wu, Y.: Multiple positive solutions of singular fractional differential system involving Stieltjes integral conditions. Electron. J. Qual. Theory Differ. Equ. 43, 1-18 (2012)

15. Klimek, M.: Sequential fractional differential equations with Hadamard derivative. Commun. Nonlinear Sci. Numer. Simul. 16, 4689-4697 (2011)

16. Ma, Q., Wang, R., Wang, J., Ma, Y.: Qualitative analysis for solutions of a certain more generalized two-dimensional fractional differential system with Hadamard derivative. Appl. Math. Comput. 257, 436-445 (2014)

17. Singh, J., Kumar, D., Baleanu, D.: New aspects of fractional Biswas-Milovic model with Mittag-Leffler law. Math. Model. Nat. Phenom. 14, 303 (2019)

18. Singh, J., Kumar, D., Baleanu, D., Rathored, S.: An efficient numerical algorithm for the fractional Drinfeld-Sokolov-Wilson equation. Appl. Math. Comput. 335, 12-24 (2018)

19. Kumar, D., Singh, J., Baleanu, D., Rathore, S.: Analysis of a fractional model of Ambartsumian equation. Eur. Phys. J. Plus 133, $259(2018)$

20. Kumar, D., Singh, J., Baleanu, D., Sushila: Analysis of regularized long-wave equation associated with a new fractional operator with Mittag-Leffler type kernel. Physica A 492, 155-167 (2018)

21. Kumar, D., Tchier, F., Singh, J., Baleanu, D.: An efficient computational technique for fractal vehicular traffic flow. Entropy 20, 259 (2018)

22. Heymans, N., Podlubny, I.: Physical interpretation of initial conditions for fractional differential equations with Riemann-Liouville fractional derivatives. Rheol. Acta 45, 765-771 (2006)

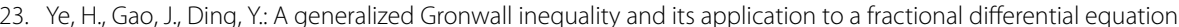
J. Math. Anal. Appl. 328, 1075-1081 (2007)

24. Gorenflo, R., Kilbas, A.A., Mainardi, F., Rogosin, S.V.: Mittag-Leffler Functions, Related Topics and Applications. Springer, Berlin (2014)

25. Cong, N.D., Doan, T.S., Siegmund, S., Tuan, H.T.: On stable manifolds for planar fractional differential equations. Appl. Math. Comput. 226, 157-168 (2014)

26. Dzherbashyan, M.M.: Integral Transforms and Representations of Functions in the Complex Plane. Nauka, Moscow (1966) (in Russian)

27. Gorenflo, R., Loutchko, J., Luchko, Y.: Computation of the Mittag-Leffler function $E_{\alpha, \beta}(z)$ and its derivative. Fract. Calc. Appl. Anal. 5(4), 491-518 (2002) Correction: Fract. Calc. Appl. Anal. 6(1), 111-112 (2003) 